\title{
Improved optogalvanic detection with voltage biased Langmuir probes
}

\author{
A. Persson, ${ }^{1}$ M. Berglund, ${ }^{2}$ and M. Salehpour ${ }^{1}$ \\ ${ }^{1}$ Department of Physics and Astronomy, Ion Physics, Uppsala University, Uppsala, SE-751 20, Sweden \\ ${ }^{2}$ Department of Engineering Sciences, Angström Space Technology Centre, Uppsala University, Uppsala, SE- \\ 75121, Sweden
}

This document is the Post-print version of a Published Work that appeared in final form in Journal of Applied Physics. It should be cited as "Anders Persson, Martin Berglund and Mehran Salehpour, Improved optogalvanic detection with voltage biased Langmuir probes, Journal of Applied Physics, vol. 116(24), p. 243301, 2015. doi.org/10.1063/1.4904964". To access the final edited and published work see: http://scitation.aip.org/content/aip/journal/jap/116/24/10.1063/1.4904964

\section{Abstract}

Optogalvanic detectors show great potential for infrared spectroscopy, especially in cavity enhanced techniques where they, in contrast to ordinary absorption detectors, can perform intracavity measurements. This enables them to utilize the signal-to-noise ratio improvement gained from the extended effective path length inside an optical cavity, without losing signal strength due to the limited amount of light exiting through the rear mirror. However, if optogalvanic detectors are to become truly competitive, their intrinsic sensitivity and stability has to be improved. This, in turn, requires a better understanding of the mechanisms behind the generation of the optogalvanic signal. The study presented here focuses on an optogalvanic detector based on a miniaturized stripline split-ring resonator plasma source equipped with Langmuir probes for detecting the optogalvanic signal. In particular, the effect of applying a constant bias voltage to one of the probes is investigated, both with respect to the sensitivity and stability, and to the mechanism behind the generation of the signal. Experiments with different bias voltages at different pressures and gas composition have been conducted. In particular, two different gas compositions (pure $\mathrm{CO}_{2}$ and $0.25 \% \mathrm{CO}_{2}$ in $99.75 \% \mathrm{~N}_{2}$ ) at six different pressures (100 $\mathrm{Pa}$ to $600 \mathrm{~Pa}$ ) have been studied. It has been shown that probe biasing effectively improves the performance of the detector, by increasing the amplitude of the signal linearly over one order of magnitude, and the stability by about $40 \%$ compared with previous studies. Furthermore, it has been shown that relatively straightforward plasma theory can be applied to interpret the mechanism behind the generation of the signal, although additional mechanisms, such as rovibrational excitation from electron-molecule collisions, become apparent in $\mathrm{CO}_{2}$ plasmas with electron energies in the 1-6 eV range. With the achieved performance improvement and the more solid theoretical framework presented here, stripline split-ring resonator optogalvanic detectors can evolve into a compact, inexpensive and easy-to-operate alternative for future infrared spectrometers. 


\section{Introduction}

Infrared (IR) spectroscopy is rapidly developing into new areas and applications following recent developments in the mid-IR laser field, primarily driven by the advent of single-mode, narrow-linewidth, tunable quantum cascade lasers (QCLs) [1]. In some applications, where the sensitivity and limit-of-detection (LOD) are of extra importance, current IR spectroscopy relies on different cavity-enhanced methods, e.g., cavity ring-down spectroscopy (CRDS) [2], intracavity absorption spectroscopy (ICAS) [3], and integrated cavity output spectroscopy (ICOS) [4]. In all these methods, the spectroscopic signal is enhanced by letting the laser beam pass repeatedly through the sample, via multiple reflections between parallel mirrors. This gives the beam a greatly increased effective path length, $L_{\text {eff }}$, through the cavity, where a decimeter long sample cell inside the cavity can receive an effective length of several kilometers, and, since the spectroscopic signal scales with $L_{\text {eff, }}$ the signal-to-noise ratio (SNR) of the system is greatly improved [3, 5]. However, a long effective path requires highly reflective cavity mirrors, and as the mirror reflectivity increases, less light exits the cavity [6]. In fact, the transmitted light intensity decreases at the same rate as $L_{\mathrm{eff}}$ increases, and, since the signal generally is measured by an IR detector outside the cavity, extended effective path lengths make it increasingly difficult to measure the signal $[3,4,6,7]$. The reduction of signal strength has to be countered by employing more advanced, expensive and complicated detectors, and, hence, the currently used signal-detection technology has become a limiting factor in the continued development of commercial cavity-enhanced IR spectroscopy [5, 7$]$.

Interesting in this respect are IR detectors based on the optogalvanic (OG) effect. Instead of studying the sample by measuring the transmitted light intensity and, consequently, the absorbance of the sample, as is customary in current IR absorption spectroscopy, an OG detector studies the sample by investigating the effect of laser-sample interactions on the impedance of a plasma inside the sample cell [8]. In the IR spectrum, the laser-sample interactions are dominated by excitations of higher order rovibrational states in the sample molecules. In such a system, the OG signal primarily stems from the collisional de-excitation of these states back to the ground level, which, in turn, affects the impedance of the plasma [8]. OG spectroscopy was a hot research topic in the late 1970 s and early 1980 s, when OG detectors were routinely used as, e.g., feedback sensors in gas lasers [8]. However, they did not find lasting applications in IR spectroscopy, mostly due to the lack of reliable light sources at relevant wavelengths at the time. When large scale application of IR spectroscopy finally started, 
in the late 1980s and early 1990s, absorption detectors quickly became dominant, due to the parallel development of new and improved detector materials, and the OG effect fell into obscurity [9]. However, since then, plasma source technology has developed too [10], and today stable and reliable plasma sources are routinely used in a wide range of different applications, spanning from microfabrication [11] to medicine [12]. Still, when attempts to re-launch OG spectroscopy have been made during the last two decades, no detector development has been attempted. Instead, old technology has been used, and these attempts failed to spark any significant scientific or commercial interest $[13,14]$.

However, from a cavity-enhanced IR spectroscopy point of view, OG detectors exhibit several great advantages that should be of interest to the field. Firstly, at its peak, OG detection often showed to be more sensitive than absorption measurements [15]. Although absorption detectors have evolved far since then, modern OG detectors, based on more stable and reliable technology, have demonstrated fast evolution and promising performance [16-18]. Secondly, and most interestingly, the fundamental differences between OG and absorption detectors make new spectrometer designs possible.

Contrary to ordinary IR detectors, OG detectors do not collect photons, but only study the interactions between the sample and the photons it absorbs, making the detector virtually transparent. This means that a welldevised OG detector can be installed inside an optical cavity without blocking the beam path [19, 20]. Inside the cavity, the laser intensity is of the same order as the incident laser beam, regardless of the reflectivity of the cavity mirrors [6]. Hence, an OG detector can, potentially, utilize the SNR improvement from an extended $L_{\text {eff, }}$ without losing signal strength due to the reduction of the transmitted laser intensity through the back mirror, i.e., it can exploit the advantages of the cavity-enhanced methods, without being affected by the main disadvantages.

In previous work, we have demonstrated the use of a miniaturized stripline split-ring resonator (SSRR) as an $\mathrm{OG}$ detector for $\mathrm{CO}_{2}$ [16-18]. The SSRR detector has been shown to be both more sensitive and reliable than current competitors, combining high reproducibility [16] with electromagnetic robustness [17]. Moreover, the volume of the sample cell of the SSRR is less than $100 \mu \mathrm{l}$, enabling analysis of extremely small samples [18]. This, in combination with the miniaturized design of the component, could enable the construction of ultracompact IR spectrometers. However, little is known about the mechanisms behind the generation of the OG effect in microwave plasmas, and one of the major objectives of this study was to investigate these further.

In an SSRR, the plasma is generated by a microwave electromagnetic field between the ends of the resonator, whereas the OG signal is measured by two Langmuir probes extending into the plasma, transversely to 
the ring structure [18]. Hence, the plasma generation is separated from the signal detection. In such a system, the OG signal relates to the laser-induced voltage change between the two probes - a situation previously investigated by, e.g., Suzuki et al. and Sekiguchi et al. [15, 21].

The study presented here, was aimed at investigating how the characteristics of the plasma - specifically the energy and density of the electrons - governed the generation of the OG signal, particularly when one of the probes was biased with a constant voltage. Moreover, the effect of such biasing on the SNR and stability of the OG signal was studied, in order to investigate if such a measurement could be used to improve the performance of the detector.

\section{Theory}

One of the most important mechanisms in OG spectroscopy is of course the excitation of the sample gas by the laser beam. In the simplest approximation, this process is assumed to be linearly dependent on the volume density of sample molecules in the excitable state, $n_{\mathrm{m}}$. The OG signal, $U_{\mathrm{OG}}$, can then be approximated by:

$$
U_{\mathrm{OG}}=n_{\mathrm{m}} I_{0} \sigma_{\mathrm{L}} \pi l r^{2} K,
$$

where $I_{0}$ is the laser intensity, $\sigma_{\mathrm{L}}$ is the absorption cross-section of the photon-molecule interaction, $\pi l r^{2}$ is the cylindrical volume in the plasma that is exposed to the laser beam, and $K$ is a proportionality parameter accounting for the effects that the physics and chemistry of the plasma have on the signal [13]. Studies have shown that $K$ is not a constant and depends on a variety of parameters [19, 20], wherefore Eq. (1) must be regarded as a poor approximation of the actual mechanisms at hand. Hence, a better understanding of how the OG signal is generated in the plasma is vital if the OG effect is to become useful in spectrometric applications. 


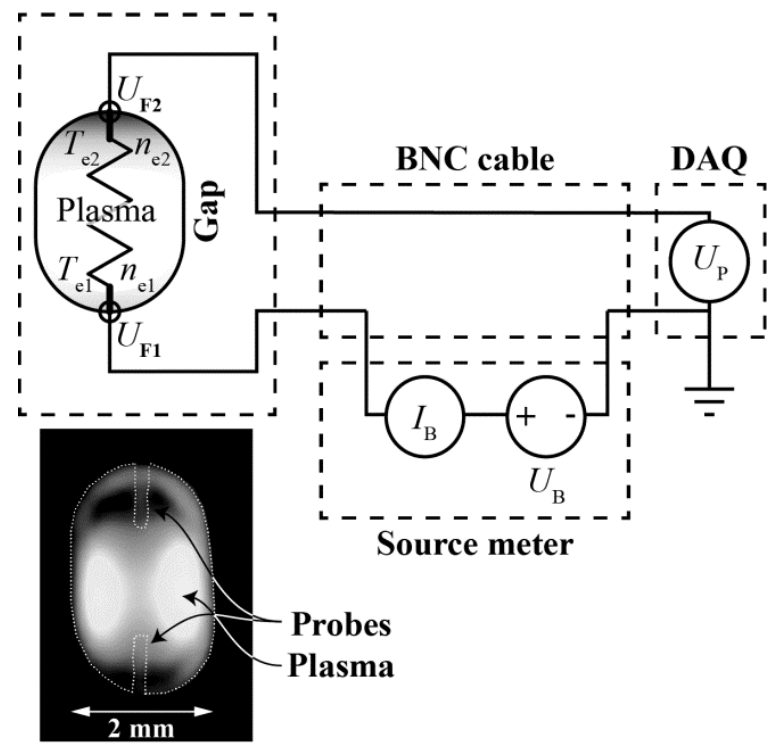

FIG. 1. Connection of the source meter to the OG signal detection circuit, where the bias voltage, $U_{\mathrm{B}}$, was applied to the shield of the BNC cable that connected the SSRR to the DAQ. The voltage over the plasma probes was measured between the inner conductor and the shield of the cable. The inset shows a photograph of an ignited SSRR, with the probes highlighted by the dotted line.

Suzuki et al. have investigated how the OG signal is generated in microwave plasmas of $\mathrm{He}, \mathrm{Ne}$ and $\mathrm{Ar}$, employing signal detection similar to the SSRR, and have shown that the signal generation is strongly dependent on asymmetries in the spatial energy and density distributions of the electrons in the plasma [15, 21]. A similar asymmetry appeared in the case of the SSRR, where the plasma was not uniformly distributed in the gap with respect to the probes, inset of Fig. 1, and, hence, $T_{\mathrm{e} 1}>T_{\mathrm{e} 2}$ was assumed [15]. In addition, Fig. 1 shows a circuit equivalent of the OG detection scheme in the SSRR. As can be seen, the measured voltage, $U_{\mathrm{P}}$, corresponded to the floating potential of probe $2, U_{\mathrm{F} 2}$, whereas probe 1 was used to bias the plasma with a voltage, $U_{\mathrm{B}}$. Using similar theory as Suzuki et al., i.e., assuming the electrons to exhibit a Maxwellian distribution, and the temperature, pressure and electric field to be at equilibrium, the floating potential, $U_{\mathrm{F}}$, of a Langmuir probe in a plasma will be defined by the plasma, $U_{\mathrm{PP}}$, and sheath, $U_{\mathrm{Sh}}$, potentials:

$$
U_{\mathrm{F}}=U_{\mathrm{PP}}+U_{\mathrm{Sh}},
$$

which are given by:

$$
\left\{\begin{array}{l}
U_{\mathrm{PP}}=\frac{k_{\mathrm{B}} T_{\mathrm{e}}}{e} \ln \left(\frac{n_{\mathrm{e}}}{n_{\mathrm{e} 0}}\right)+U_{\mathrm{PP} 0} \\
U_{\mathrm{Sh}}=\frac{k_{\mathrm{B}} \alpha T_{\mathrm{e}}}{e}
\end{array} .\right.
$$

where $U_{\mathrm{PP} 0}$ is the unperturbed plasma potential, $k_{\mathrm{B}}$ is Boltzmann's constant, $T_{\mathrm{e}}$ is the electron temperature, $e$ is the charge of an electron, and $n_{\mathrm{e}}$ and $n_{\mathrm{e} 0}$ are the electron densities of the perturbed and the unperturbed plasma, respectively. The sheath potential is the potential difference between $U_{\mathrm{PP}}$ and the floating probe surface, and is 
defined by the constant $\alpha$, which is determined by the probe geometry, and electron and ion masses, $m$ and $M$, so that $\alpha=0.5 \ln \left[\pi m(2 M)^{-1}\right]$ for a cylindrical probe $[15,21]$. Hence, $\alpha$ assumes negative values. Consequently, the measured voltage corresponds to:

$$
U_{\mathrm{P}}=U_{\mathrm{F} 2}=\frac{k_{\mathrm{B}} T_{\mathrm{e} 2}}{e}\left[\ln \left(\frac{n_{\mathrm{e} 2}}{n_{\mathrm{e} 0}}\right)+\alpha\right]+U_{\mathrm{PP} 0}
$$

where subscript 2 refers to the conditions at probe 2, i.e., the floating probe.

The OG signal, $U_{\mathrm{OG}}$, corresponded to a laser-induced perturbation of $U_{\mathrm{P}}$, i.e. $U_{\mathrm{OG}}=\delta U_{\mathrm{P}}$. In terms of the plasma, such a perturbation correlated to a perturbation of $T_{\mathrm{e}}$ and/or $n_{\mathrm{e}}$, but since the low-energy IR photons of the laser could be expected to only excite rovibrational transitions in the target molecules and not alter their electronic states [8], it was assumed that temperature perturbations, $\delta T_{\mathrm{e}}$, dominated over density perturbations, $\delta n_{\mathrm{e}}$, i.e., $\delta T_{\mathrm{e}} / T_{\mathrm{e}}>>\delta n_{\mathrm{e}} / n_{\mathrm{e}}$ [22]. Assuming the perturbations to be small, i.e. $\delta U_{\mathrm{P}}<<U_{\mathrm{P}}$ and $\delta T_{\mathrm{e}}<<T_{\mathrm{e}}$, their mutual relationship can be approximated by:

$$
\frac{\delta U_{\mathrm{P}}}{U_{\mathrm{P}}} \sim \frac{\delta T_{\mathrm{e}}}{T_{\mathrm{e}}} .
$$

Previous studies [16] have shown that these approximations are valid in the typical operational region of the SSRR, where $\delta T_{\mathrm{e}} / T_{\mathrm{e}} \approx \delta U_{\mathrm{P}} / U_{\mathrm{P}} \approx 1 / 25\left(h c \lambda^{-1} \approx 0.12 \mathrm{eV}\right.$ for $\left.\lambda=10.59 \mu \mathrm{m}\right)$. Finally, the relationship between $U_{\mathrm{OG}}$ and $\delta T_{\mathrm{e}}$ can be calculated from Eq. (5) yielding:

$$
U_{\mathrm{OG}} \sim U_{\mathrm{P}} \frac{\delta T_{\mathrm{e} 2}}{T_{\mathrm{e} 2}}
$$

However, it should be pointed out that these expressions contain significant simplifications, especially since the SSRR typically operates close to the transition between when collisionless sheaths can be assumed, and collisional sheath theory starts to apply. Hence, the theory presented in this study is not intended for absolute calculations, but only for relative comparisons. 


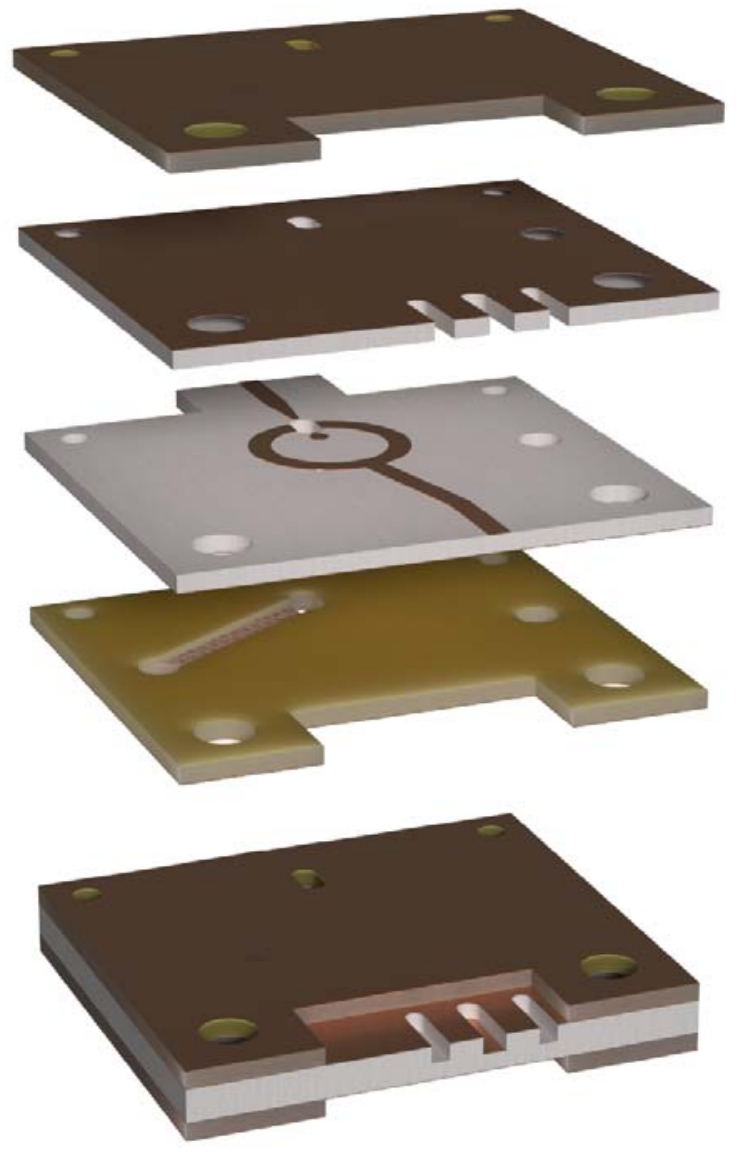

FIG. 2.Exploded view or the SSRR concept on top of a non-exploded device.

\section{Materials and Methods}

The design of the SSRR used in this study, Fig. 2, was almost identical to the one in [16], having a 2 mm wide gap and a resonance frequency of $2.7 \mathrm{GHz}$, although the windows were replaced by $1 / 2$ inch antireflection coated

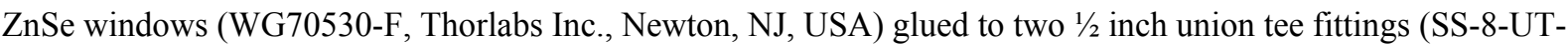
3, Swagelok, Solon, OH, USA), which served as gas connectors, instead of the integrated channels. The electronics, gas handling system, and optics of the setup too resembled that of [16], where the laser beam from a single longitudinal mode ${ }^{12} \mathrm{CO}_{2}$ laser was modulated by a chopper before being coupled through the reference and SSRR cells to a power meter. However, there were a few differences compared with the earlier setup, Fig. 3. Firstly, a discrete pyroelectric power meter (Model QS-I-Test GenTech-EO, Quebec, QC, Canada) was installed behind the SSRR, in parallel with the previously used thermopile power meter, to study the dynamics of the laser pulses. Secondly, a general purpose source meter (Model 2400, Keithley Instruments Inc., OH, USA) was connected to the signal detection side of the SSRR, to allow constant voltage biasing of the plasma probes, and I$\mathrm{V}$ curve measurements of the plasma in parallel with the $\mathrm{OG}$ detection. Moreover, the ${ }^{13} \mathrm{CO}_{2}$ laser was not used in these experiments. 


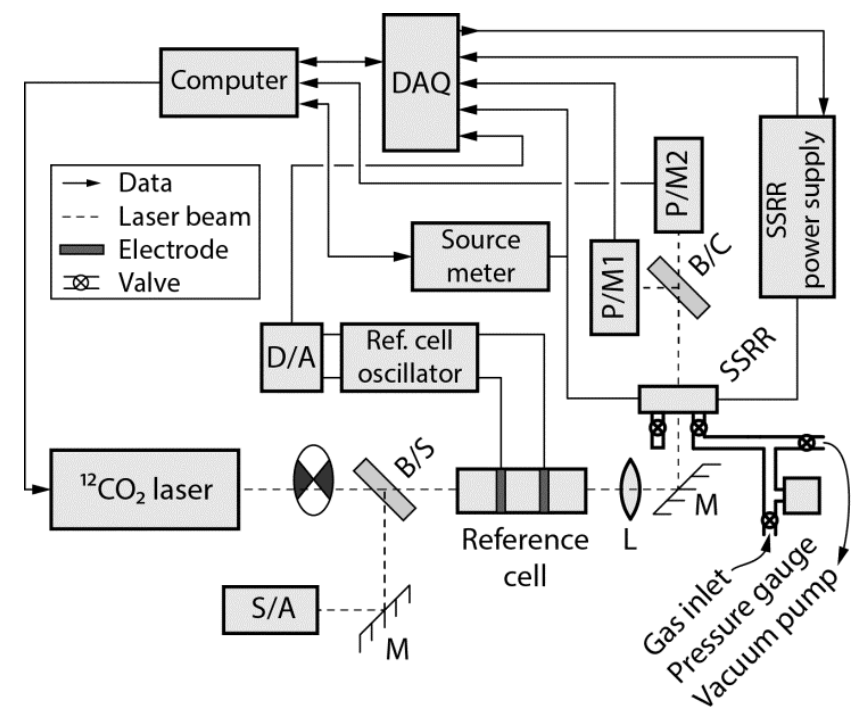

FIG. 3. Schematics of the OG spectroscopy setup including optics, gas handling and electronics. Here, $B / S$ denotes beam splitter, $C$ chopper, $D / A$ differential amplifier, $D A Q$ data acquisition, $L$ lens, $M$ mirror, $S / A$ spectrum analyzer, and $P / M 1$ and $P / M 2$ denote the pyroelectric and thermopile power meters, respectively.

The source meter was connected to the shield of the coaxial cable, connecting the SSRR to the DAQ, Fig. 1. The connection was made via a custom-made electromagnetically shielded box, to minimize noise pick-up in the detection circuit. Hence, the voltage at probe $2, U_{\mathrm{P}}$, could be measured while applying a constant bias voltage, $U_{\mathrm{B}}$, to probe 1 (Fig. 1). The source meter also measured the current through the plasma, $I_{\mathrm{B}}$, hence, enabling measurements of the plasma's I-V characteristics. The I-V curves were used to calculate the plasma potential, $U_{\mathrm{PP}}$, and electron temperature, $T_{\mathrm{e}}$, in the same way as in [17] assuming collisionless, un-magnetized plasma conditions [23].

The experiments focused on investigating the dependence of the OG signal on pressure, gas composition and, particularly, the probe bias voltage. Two different gas compositions - pure $\mathrm{CO}_{2}$ and $0.25 \% \mathrm{CO}_{2}$ in $99.75 \%$ $\mathrm{N}_{2}$ - were studied at six different pressures ranging from 100 to $600 \mathrm{~Pa}$. The $\mathrm{CO}_{2}-\mathrm{N}_{2}$ gas mixture was created by 1.) filling the system with $100 \mathrm{~Pa}$ of pure $\mathrm{CO}_{2}$, 2.) adding $1900 \mathrm{~Pa}$ of $\mathrm{N}_{2}$, 3.) pumping the system to $100 \mathrm{~Pa}, 4$.) adding $1900 \mathrm{~Pa}$ of $\mathrm{N}_{2}$, and 5.) pumping the system to the desired pressure.

Before each measurement, the spectrum around the P20 transition of ${ }^{12} \mathrm{C}^{16} \mathrm{O}_{2}(\lambda=10.6 \mu \mathrm{m})$ was recorded, by tuning the laser wavelength with the piezoelectric control of the laser cavity [16]. These spectra were later used to estimate the gas temperature in the sample cell. The laser was then centered and stabilized at the peak of the P20 transition. During a measurement, the bias voltage was swept in steps of $2.5 \mathrm{~V}$, back and forth from 60 to $-60 \mathrm{~V}$, twice, while recording $U_{\mathrm{OG}}, U_{\mathrm{P}}$, and $I_{\mathrm{B}}$. In addition, the $\mathrm{OG}$ signals from the sample and reference cells and the mean potential of probe 2, the laser power from the two power meters and the reflected microwave power from the SSRR were recorded. The sampling time in each step was $5 \mathrm{~s}$, meaning that a total of $20 \mathrm{~s}$ of 
data, recorded at four separate occasions, were gathered for each $U_{\mathrm{B}}$. Throughout the measurements, the modulation frequency of the laser was kept constant at $40 \mathrm{~Hz}$. In the experiments with the $\mathrm{CO}_{2}-\mathrm{N}_{2}$ mixture, the SSRR was driven with slightly higher power $(2.5 \mathrm{~dB})$ than when studying pure $\mathrm{CO}_{2}$, to assure stable plasma conditions at all pressures and biases. The different parameters of the experiments are summarized in Tab. 1.

TAB 1. Gas mixture, laser transition, modulation frequency, attenuation, pressure and sampling time of the main experiments

\begin{tabular}{ccccccc}
\hline Experiment & Gas & Laser transition & $f_{\mathrm{C}}[\mathrm{Hz}]$ & Att. [dB] & Pressure [Pa] & Sampling time [s] \\
\hline 1 & $0.25 \% \mathrm{CO}_{2}$ in $\mathrm{N}_{2}$ & $\mathrm{P} 20$ & 40 & 0 & $100-600$ & 5 \\
2 & $\mathrm{CO}_{2}$ & $\mathrm{P} 20$ & 40 & 2.5 & $100-600$ & 5 \\
\hline
\end{tabular}

In addition, the stability of the signal was studied in a separate experiment where $200 \mathrm{~s}$ of data was collected at two different pressures (200 Pa and $500 \mathrm{~Pa})$, and four different bias voltages $( \pm 25$ and $\pm 50 \mathrm{~V})$. The data series were used to calculate the overlapped Allan variance, using the Matlab (Matlab 2012b, The Mathworks Inc., Natick, MA, USA) program “allan v1.71“ [24].

\section{Results}

The dependence of the OG signal, probe potential and plasma current on the bias voltage, i.e., of $U_{\mathrm{OG}}, U_{\mathrm{P}}$, and $I_{\mathrm{B}}$ on $U_{\mathrm{B}}$, was similar in all the experiments, where both $U_{\mathrm{OG}}$ and $U_{\mathrm{P}}$ started to increase after $U_{\mathrm{B}}$ had passed the floating potential, and increased linearly after $U_{\mathrm{B}}$ had passed the plasma potential, Fig. $4 . I_{\mathrm{B}}$ on the other hand, behaved as expected from Langmuir probe theory, and the I-V curves showed distinct electron and ion regions at sufficiently positive and negative probe bias, Fig. 4. The $U_{\mathrm{B}}$ sweeps showed some hysteresis, likely due to charging of the probes, which caused some uncertainty, particularly in the I-V curves (as seen by the error bars), but the results were stable and reliable, and could be reproduced with around $1 \%$ precision over several days. The stability of the laser was monitored throughout the experiments, by measuring the response of the power meters and the reference cell. All measurements with noticeable laser drift were repeated, although this was a dismissible problem, since the laser typically remained stable up to $1 \mathrm{~h}$ after proper stabilization. Finally, in order to account for short time-scale variations, such as drift in the chopper frequency, the OG signal from the SSRR was scaled by the OG signal from the reference cell. 


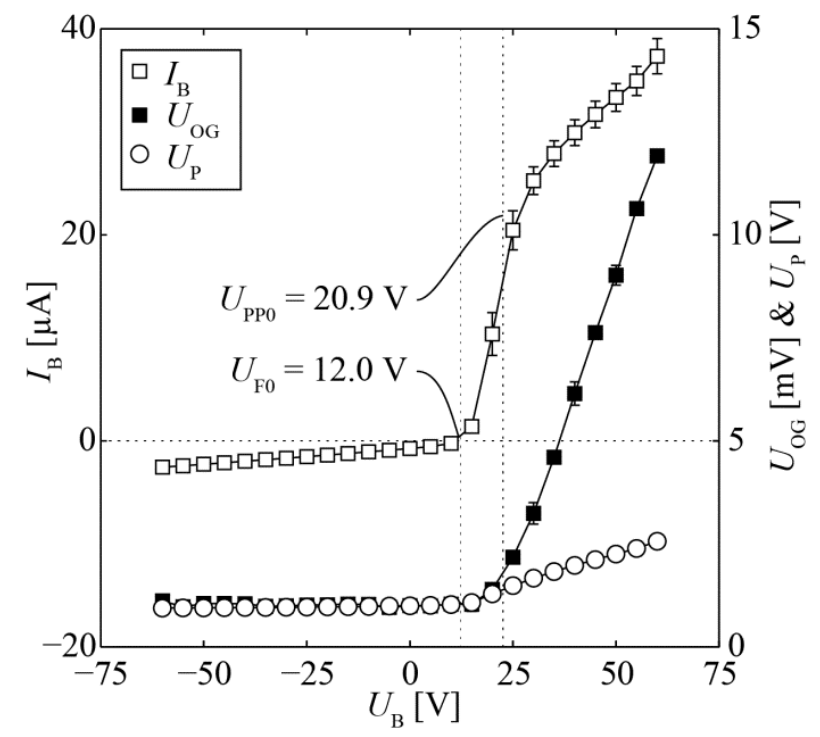

FIG. 4. Langmuir curve of a pure $\mathrm{CO}_{2}$ plasma at $600 \mathrm{~Pa}$ (white squares), and corresponding change in OG signal (black squares) and $U_{\mathrm{P}}$ (white circles). The right y axis is scaled in $\mathrm{mV}$ for $U_{\mathrm{OG}}$ and $\mathrm{V}$ for $U_{\mathrm{P}}$.

The stability measurements yielded stable OG responses at positive $U_{\mathrm{B}}$, where the SNR typically improved linearly as $U_{\mathrm{B}}$ increased, Fig. 5. However, from preliminary studies, it was found that sufficiently high bias voltages $(>55 \mathrm{~V})$ started to affect the both the stability and the noise of the plasma, until the plasma went out at roughly $U_{\mathrm{B}}>65 \mathrm{~V}$ depending on, e.g., the pressure. The OG signal at low bias voltages, on the other hand, was highly unstable, showing clear signs of drift, Fig. 5. However, high negative bias voltages $(<-70 \mathrm{~V})$ did not quench the plasma.

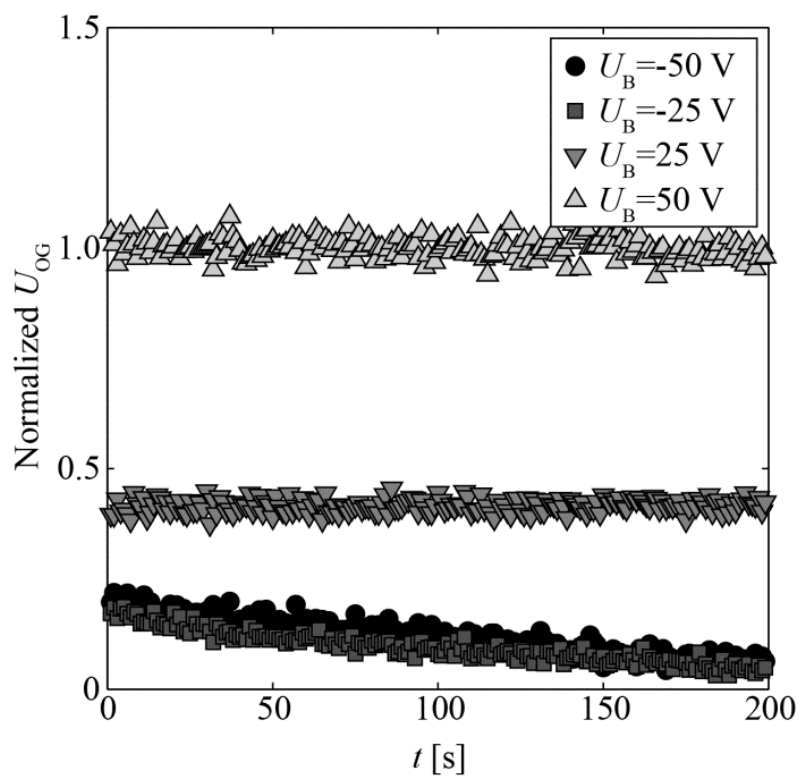

FIG. 5 Stability of $U_{\mathrm{OG}}$ at different $U_{\mathrm{B}}$ in a $500 \mathrm{~Pa} \mathrm{CO}_{2}$ plasma. Here, $U_{\mathrm{OG}}$ was normalized to the mean of the $U_{\mathrm{B}}=50 \mathrm{~V}$ measurement. 


\section{Discussion}

An initial observation was that probe biasing clearly constituted an effective method of improving the performance of the SSRR detector, where both the amplitude and stability of the signal was considerably improved with increasing bias voltage, Figs. 4 and 5. Although these results were impressive by themselves, a better understanding of the generation of the OG signal was still necessary in order to reap the benefits in a spectrometric application.

Hence, the focus of the study was to investigate the characteristics of the plasma, and how the biasing of the probes affected the mechanism behind the generation of the OG signal, especially in the region where $U_{\mathrm{B}}>U_{\mathrm{PP} 0}$ and both $U_{\mathrm{OG}}\left(U_{\mathrm{B}}\right)$ and $U_{\mathrm{P}}\left(U_{\mathrm{B}}\right)$ were increasing linearly, Fig. 4. The data in this region was postprocessed, to calculate the slopes of the $U_{\mathrm{OG}}\left(U_{\mathrm{B}}\right)$ and $U_{\mathrm{P}}\left(U_{\mathrm{B}}\right)$ curves above the plasma potential. Curve fitting was performed in Matlab, and, similarly, $U_{\mathrm{PP} 0}$ and $T_{\mathrm{e} 0}$ (where subscript 0 indicates the plasma parameters of the unperturbed plasma) were calculated from the I-V curves using similar theory as in [17]. However, the electron and ion densities, which too could have been estimated from the I-V curves, were omitted, since such a calculation would have required a reliable approximation of the effective surface area of the floating probe. The fact that the collection ability of the probes depend strongly on such surface conditions may affect other parameters as well. Hence, efforts to characterize such effects and to develop micro-probes with a more welldefined surface are currently being made in our laboratory.
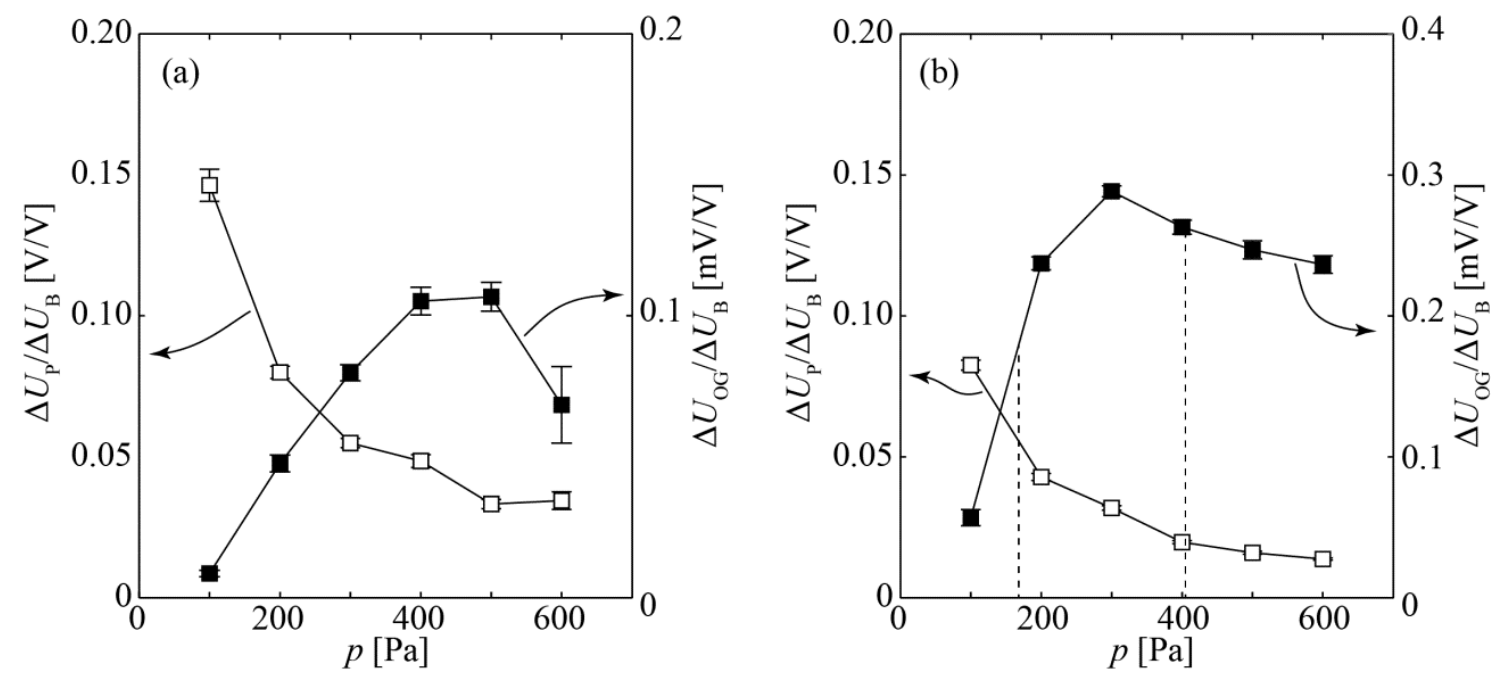

FIG. 6. First derivative of $U_{\mathrm{P}}$ (white squares) and the OG signal (black squares) in the linear part of the $U_{\mathrm{B}}$ sweep, for gas mixes of $0.25 \% \mathrm{CO}_{2}$ in $\mathrm{N}_{2}$ (a) and pure $\mathrm{CO}_{2}$ (b) at different pressures. The dashed lines in (b) correspond to the gray areas in Fig. 7 (b). 

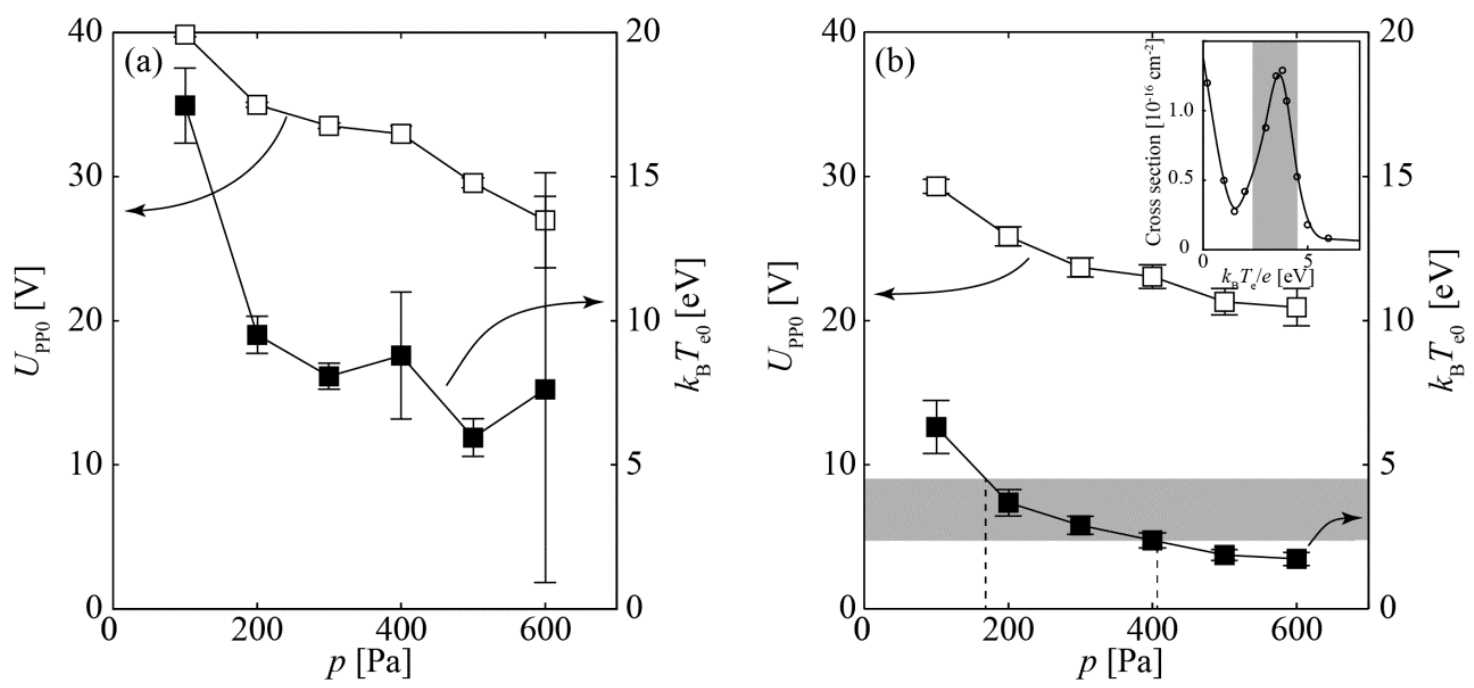

FIG. 7. Plasma potential, $U_{\mathrm{PP}}$ (white squares), and electron temperature $k_{\mathrm{B}} T$, (black squares), for gas mixes of $0.25 \% \mathrm{CO}_{2}$ in $\mathrm{N}_{2}$ (a) and pure $\mathrm{CO}_{2}$ (b) at different pressures. The inset shows the vibrational excitation cross section of the first symmetric stretch mode (100) of $\mathrm{CO}_{2}$ from collisions with electrons [25]. Gray areas in both (b) and the inset enclose the cross-section peak around $3 \mathrm{eV}$, and dashed lines show the corresponding pressure range.

Figures 6 and 7 show the resulting parameters for the different pressures of the experiments. However, in order to compare them with the theoretical predictions, some additional derivation was necessary to understand the impact of increasing $U_{\mathrm{B}}$ on $U_{\mathrm{P}}$ and $U_{\mathrm{OG}}$. Firstly, when $U_{\mathrm{B}}$ approached the plasma potential of the unbiased plasma, $U_{\mathrm{PP} 0}$, probe 1 started to drain the plasma so that $U_{\mathrm{PP}}$ had to rise above $U_{\mathrm{B}}$ [26]. Beyond $U_{\mathrm{PP} 0}$, the potential of probe 1 effectively defined the plasma potential, as described in Fig. 8. Although the actual plasma potential would be slightly higher than the bias voltage, $U_{\mathrm{PP}} \approx U_{\mathrm{B}}$ for $U_{\mathrm{B}}>U_{\mathrm{PP} 0}$ was assumed for simplicity. Secondly, Fig. 4 shows that the floating potential at probe 2, i.e. $U_{\mathrm{P}}$, increased more slowly than $U_{\mathrm{PP}}$ with respect to $U_{\mathrm{B}}$, Fig. 8. According to Eqs. (2-4), this means that the sheath potential had to be linearly decreasing with respect to $U_{\mathrm{B}}$ in this interval - a fact that is further clarified in Fig. 8, where the effect of an increasing sheath potential on the floating potential of the unbiased probe is illustrated. Applying a linear curve passing through $U_{\mathrm{Sh} 0}$ at $U_{\mathrm{B}}=U_{\mathrm{PP} 0}, U_{\mathrm{Sh}}=U_{\mathrm{Sh} 0} U_{\mathrm{PP} 0}{ }^{-1} U_{\mathrm{B}}$ was assumed for $U_{\mathrm{B}}>U_{\mathrm{PP} 0}$. 


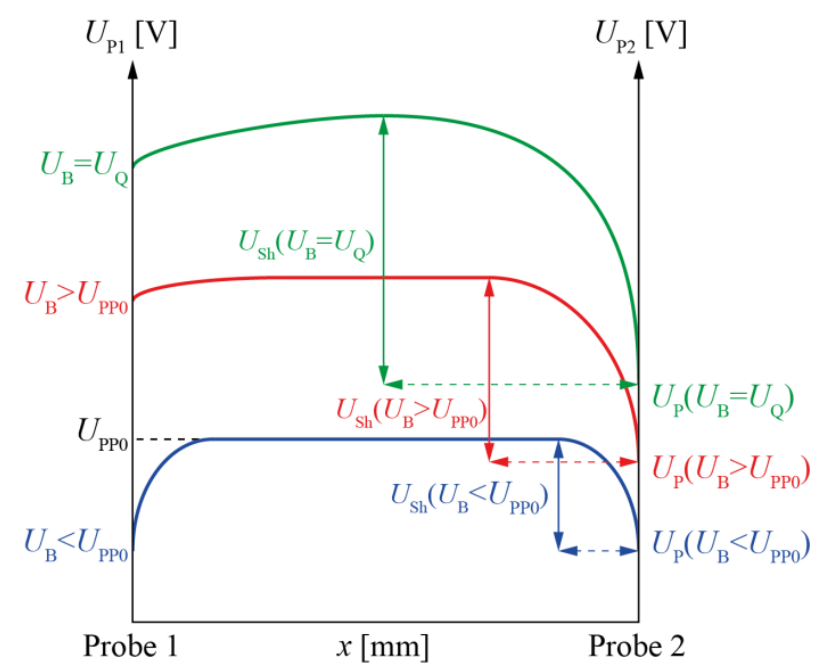

Fig. 8. Illustration of the potential distribution in the plasma at three different probe biases, where the two y axes indicate the voltages at the two probes. The dashed lines indicate the effect of an increasing probe bias on the strength and width of the sheath at the unbiased probe.

Regarding the OG effect, Eq. (1) implies that $\delta T_{\mathrm{e}}$ in Eq. (6) is primarily dependent on $n_{\mathrm{m}}$, since the number of molecules was the measured entity in measurements with a fixed laser power and wavelength, and, assuming ideal gas conditions, $\delta T_{\mathrm{e}} \sim p / T_{\mathrm{G}}$, where $p$ is the pressure and $T_{\mathrm{G}}$ is the gas temperature. The latter was estimated from Cauchy-Lorentz distribution fits to the recorded spectra of the P20 peak. An example of this can be seen in Fig. 9. The distribution of the P20 peak could be assumed to follow a Cauchy-Lorentz distribution if molecular collisions were the main cause of the broadening of the peak [27]. Collisional broadening becomes dominant as the pressure rises; however, at the pressures of the experiments described here, a convolution between a CauchyLorentz and a Gaussian distribution, known as a Voigt profile, would probably have been a better estimation, since Doppler broadening, too, will affect the distribution of the peak [19]. Still, to be able to extract the gas temperature, the Cauchy-Lorentz distribution was deemed adequate, keeping the greater uncertainty at lower pressures in mind.

The Cauchy-Lorentz distribution is defined by the half width at half maximum of the broadening, $\gamma$, which could be extracted from the curve fits [27]. Again assuming ideal gas conditions, it can be shown that $\gamma \sim p T_{G}^{-0.5}$, and, thus, $T_{\mathrm{G}} \sim p^{2} \gamma^{-2}$ and $n_{\mathrm{m}} \sim \gamma^{2} p^{-1}$. The results showed a linear dependence of $T_{\mathrm{G}}$ on $p$ in both experiments $\left(R^{2}=0.999\right.$ for pure $\mathrm{CO}_{2}$ and $R^{2}=0.970$ for $\mathrm{CO}_{2}$ in $\left.\mathrm{N}_{2}\right)$, which was in agreement with previous studies of similar plasma sources [28]. However, as can be seen in the inset of Fig. 9, the results for the $100 \mathrm{~Pa}$ experiments deviated considerably from the common trend, probably due to a combination of the Doppler broadening becoming more dominant and reduced SNR. Hence, the gas temperatures of the $100 \mathrm{~Pa}$ experiments were regarded as outliers in the subsequent calculations. 


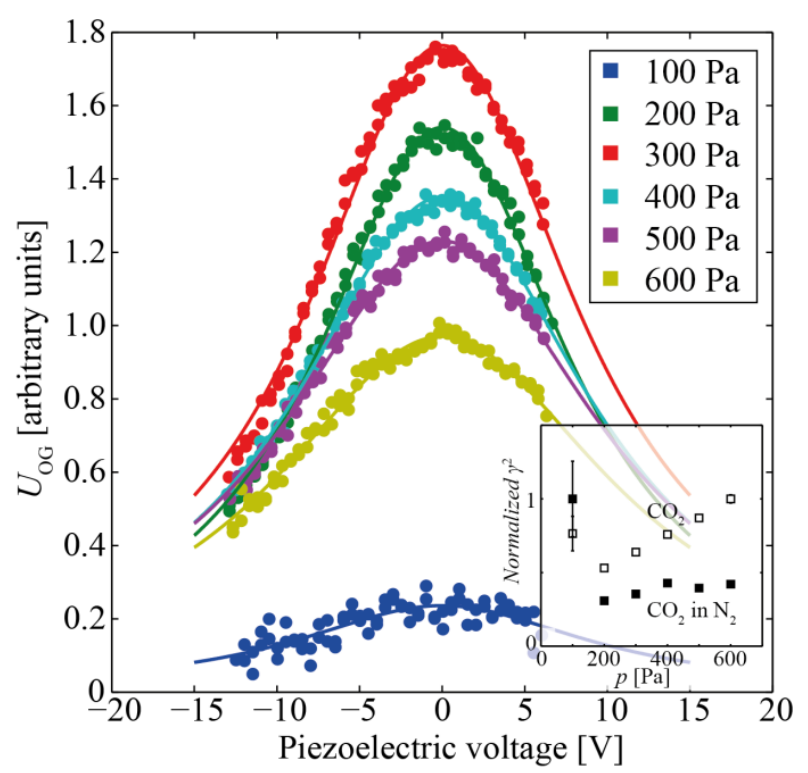

FIG. 9. Spectral distribution of the $\mathrm{P} 20$ peak in pure $\mathrm{CO}_{2}$ (circles) along with the Cauchy-Lorentz distribution fits (lines). The inset shows the dependence of the estimated gas temperature on pressure.

These observations and asumptions, along with Eqs. (4) and (6), yield new expressions for the different potentials in the region where $U_{\mathrm{B}}>U_{\mathrm{PP} 0}$ :

$$
\left\{\begin{array}{l}
U_{\mathrm{PP}} \approx U_{\mathrm{B}} \\
U_{\mathrm{Sh}} \approx \frac{U_{\mathrm{Sh} 0}}{U_{\mathrm{PP} 0}} U_{\mathrm{B}} \\
U_{\mathrm{P}} \sim\left(1+\frac{U_{\mathrm{Sh} 0}}{U_{\mathrm{PP} 0}}\right) U_{\mathrm{B}}=\left(1+\frac{\alpha k_{\mathrm{B}} T_{\mathrm{e} 0}}{e U_{\mathrm{PP} 0}}\right) U_{\mathrm{B}} \\
U_{\mathrm{OG}} \sim\left(1+\frac{U_{\mathrm{Sh} 0}}{U_{\mathrm{PP} 0}}\right) U_{\mathrm{B}} \frac{\delta T_{e}}{T_{e 0}} \sim\left(\frac{e}{k_{\mathrm{B}} T_{\mathrm{e} 0}}+\frac{\alpha}{U_{\mathrm{PPO}}}\right) \frac{\gamma^{2}}{p} U_{\mathrm{B}}
\end{array}\right.
$$

From a plasma point of view, the dependence of $U_{\mathrm{PP}}$ and $U_{\mathrm{Sh}}$ on $U_{\mathrm{B}}$ stems from the mechanism behind the collection of electrons by the probes at $U_{\mathrm{B}}>U_{\mathrm{PP} 0}$. In this region, the biased probe will collect slow-moving electrons more easily than faster ones, based on orbital motion theory, causing an indirect heating of the plasma. This affects both the energy and density of the electrons, and the relationship between these two parameters becomes defined by the restriction of the first term of Eq. (7), where $T_{\mathrm{e}} \sim \ln \left(n_{\mathrm{e}}\right)^{-1}$ enables $U_{\text {PP }}$ to follow $U_{\mathrm{B}}$, Fig. 8 . Hence, Eq. (7) can only be assumed to be valid as long as the plasma is able to sustain itself with electrons from secondary electron sources such as the cell walls. Beyond this point, the bias voltage will start to quench the plasma, which will eventually go out when the Debye length approaches the dimensions of the plasma cell. The growing Debye length, roughly corresponding to the width of the sheath at the probes, is illustrated in Fig. 8 . Such quenching is most likely the cause of the plasma perturbations observed at high bias voltages, and possibly of the uncertainty in the $600 \mathrm{~Pa}$ measurement in experiment 1, Figs. 6 (a) and 7 (a). Hence, Eq. (7) should rather 
be defined in an interval of $U_{\mathrm{PP} 0}<U_{\mathrm{B}}<U_{\mathrm{Q}}$, where $U_{\mathrm{Q}}$ corresponds to the voltage where the quenching process starts to become apparent.

From Eq. (7), the first order derivatives of $U_{\mathrm{P}}$ and $U_{\mathrm{OG}}$ with respect to $U_{\mathrm{B}}$ could be calculated:

$$
\left\{\begin{array}{l}
\frac{\partial U_{\mathrm{P}}}{\partial U_{\mathrm{B}}} \sim\left(1+\frac{\alpha k_{\mathrm{B}} T_{\mathrm{e} 0}}{e U_{\mathrm{PP} 0}}\right) \\
\frac{\partial U_{\mathrm{OG}}}{\partial U_{\mathrm{B}}} \sim\left(\frac{e}{k_{\mathrm{B}} T_{\mathrm{e} 0}}+\frac{\alpha}{U_{\mathrm{PP} 0}}\right) \frac{\gamma^{2}}{p} \text { for } U_{\mathrm{PP} 0}<U_{\mathrm{B}}<U_{\mathrm{Q}} .
\end{array}\right.
$$

In order to compare the predictions of Eq. (8) with the measurements in Figs. 6 and 7, linear curve fits were made to the data, as described in Tab. 2. The curve fitting was performed using the weighted total least-squares algorithm presented in [29]. The goodness of the fits was evaluated with Pearson's $X^{2}$ test with York's weights. The major benefit of this evaluation method was the fact that it took the uncertainty in both coordinates into account. Hence, measurements with greater uncertainty, e.g., the $400 \mathrm{~Pa}$ and $600 \mathrm{~Pa}$ measurements in Fig. 7 (a), could be included. With six degrees of freedom, a $X^{2} \leq 1.63$ corresponded to a probability of more than $95 \%$ that the measured data agreed with the model.

TAB. 2. Goodness of fits of data from Figs. 6 and 7 to Eq. (8), evaluated with Pearson's $X^{2}$ test with York's weights.

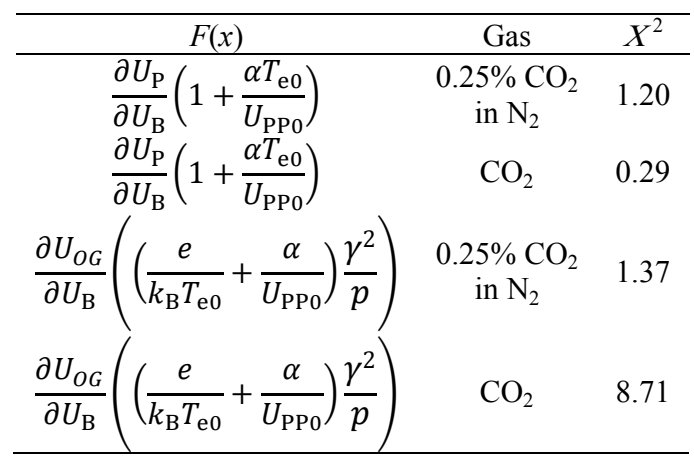

As can be seen in Tab. 2, the agreement between the experiments and the model was generally good, with one major exception. In the case of $U_{\mathrm{OG}}$ in pure $\mathrm{CO}_{2}$, the experimental results clearly deviated from the predicted behavior, indicating that an additional mechanism was active in this case. Furthermore, the fact that $U_{\mathrm{P}}$ in pure $\mathrm{CO}_{2}$ still agreed well with the model, indicated that this extra mechanism primarily affected the parameter that was unique to $U_{\mathrm{OG}}$, namely $\delta T_{\mathrm{e}}$.

Equation (1) states that the OG signal, and consequently $\delta T_{\mathrm{e}}$, is dependent on the number of excitable molecules in the system. In the system investigated here, these were $\mathrm{CO}_{2}$ molecules in either the first symmetric or asymmetric stretch modes, which can be subjected to either absorption or stimulated emission in the interaction with the laser beam. Of these two processes, absorption heats and stimulated emission cools the 
plasma, and, since the OG signal was in phase with the modulated laser beam in both experiments, it can be assumed that absorption was the prevailing process behind the generation of the signal throughout this study. Hence, the relative strength of the signal was dependent on the number of $\mathrm{CO}_{2}$ molecules in the first asymmetric stretch mode, commonly abbreviated (100).

The number of $\mathrm{CO}_{2}$ molecules in the (100) state is primarily governed by thermal activation from the ground state [30]. In a plasma, however, additional complexity is added by vibrational excitation from collisions between $\mathrm{CO}_{2}$ molecules and electrons. The inset of Fig. 7 shows the cross section of such collisions resulting in vibrational excitation to the (100) state [25]. As can be seen, the collisional cross section shows a peak at roughly $3 \mathrm{eV}$, and, interestingly, this peak correlated to the region where the behavior of the $\mathrm{OG}$ signal from pure $\mathrm{CO}_{2}$ deviated from the predictions of the model, as indicated by the gray areas in Fig. 7. Similarly, a clear correlation between the amplitude of OG signal and the electron excitation of the (100) state was found when studying the OG signal from pure $\mathrm{CO}_{2}$ at the floating potential of the plasma, i.e., $U_{\mathrm{OG}}\left(U_{\mathrm{B}}=U_{\mathrm{F} 0}\right)$, Fig. 10. Here, the signal at the floating potential was chosen to compare the different experiments at similar plasma conditions.

Clearly, electron collisions constituted an important process in the generation of the OG signal in pure $\mathrm{CO}_{2}$. Similar results would probably have been found in the case of $\mathrm{CO}_{2}-\mathrm{N}_{2}$ mixtures, if the electron temperature had been somewhat lower, since electrons in the $2-3 \mathrm{eV}$ range effectively excite $\mathrm{N}_{2}$ into the first vibrational $(v=1)$ state [31]. This state is in close resonance with the first asymmetric stretch mode of $\mathrm{CO}_{2}-$ the (001) mode. This resonance promotes increased population of the (001) level and, consequently, stimulated emissions from the sample [30]. $\mathrm{N}_{2}$ excitation of $\mathrm{CO}_{2}$ is routinely utilized to populate the upper laser level in a $\mathrm{CO}_{2}$ laser, and would have been observed as an apparent cooling of the plasma in experiment 1. However, at $T_{\mathrm{e} 0}>5 \mathrm{eV}$, the vibrational excitation of $\mathrm{N}_{2}$ from collisions with electrons is fairly ineffective [31], and, hence, $\delta T_{\mathrm{e} 0}$ remained more or less independent of $T_{\mathrm{e} 0}$ in experiment 1 . This is most probably the reason why the proposed theoretical model applied to the generation of the OG signal in $\mathrm{CO}_{2}-\mathrm{N}_{2}$ mixtures, but not in pure $\mathrm{CO}_{2}$. It can also be assumed that the model would apply to a hotter pure $\mathrm{CO}_{2}$ plasma $\left(T_{\mathrm{e} 0}>7 \mathrm{eV}\right)$, since the collisional excitation of the (100) level levels out at higher electron temperatures, Fig. 10.

It should also be mentioned that electron collisions may have additional effects of the generation of the OG signal, e.g., by dissociative attachment of $\mathrm{CO}_{2}$ [25], which obviously will affect the number of excitable molecules in the plasma. We have previously shown that a good understanding of the chemistry of the plasma is crucial for the interpretation of the OG signal [19], and such effects will have to be studied further. 


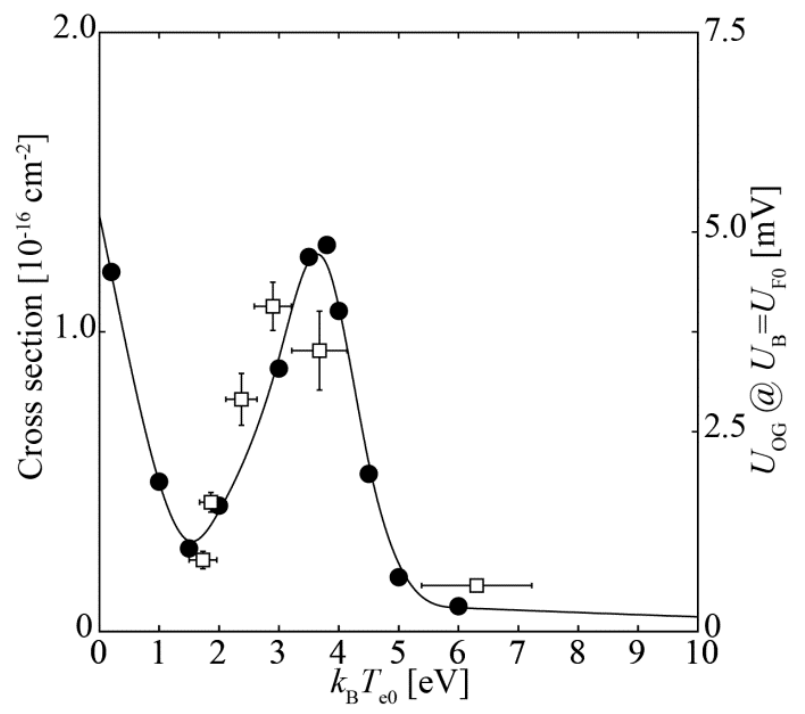

FIG. 10. Measured OG signal at the floating potential (white squares) and the electron collisional cross-section of vibrational excitation of the first symmetric stretch mode (100) of $\mathrm{CO}_{2}$ from [25] (filled circles) as a function of electron temperature.

Turning the attention to the relationship between the OG signal and the probe potential, i.e., $U_{\mathrm{OG}}$ and $U_{\mathrm{P}}$, Eq. (6) indicates a somewhat less complicated situation, where the OG signal primarily depends on the electron temperature. Figure 11 shows $U_{\mathrm{OG}}$ as a function of $U_{\mathrm{P}}$ in the two experiments. Similar to Fig. $4, U_{\mathrm{OG}}$ increased linearly at sufficiently high $U_{\mathrm{P}}$, more precisely beyond a $U_{\mathrm{P}}\left(U_{\mathrm{B}}\right)$ where $U_{\mathrm{B}}>U_{\mathrm{PP} 0}$. Again, the slopes of these curves were calculated by curve fitting in Matlab, and were compared to the proposed model by applying the same assumptions as in Eq. (8) to Eq. (6), i.e.:

$$
\frac{\partial U_{\mathrm{OG}}}{\partial U_{\mathrm{P}}} \sim \frac{\gamma^{2}}{p T_{\mathrm{e} 0}}, \text { for } U_{\mathrm{PP} 0}<U_{\mathrm{B}}<U_{\mathrm{Q}}
$$
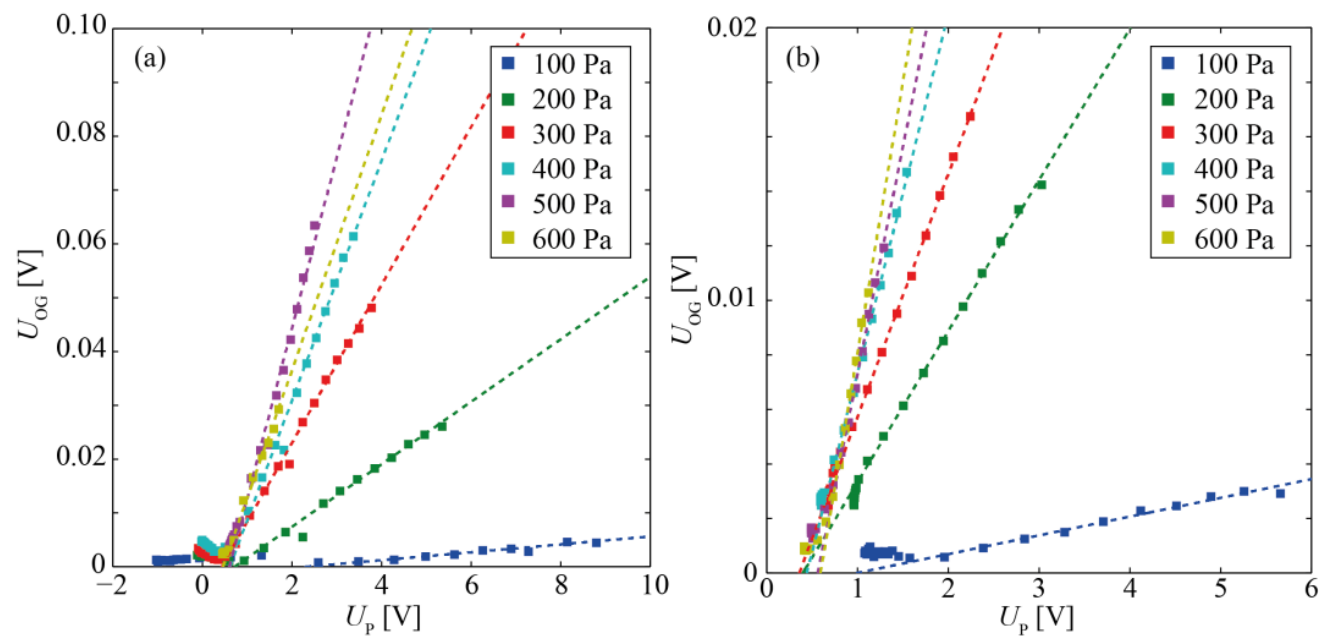

FIG. 11. Relationship between the OG signal and $U_{\mathrm{P}}$ at different pressures, for gas mixtures of $0.25 \% \mathrm{CO}_{2}$ in $\mathrm{N}_{2}$ (a) and pure $\mathrm{CO}_{2}(\mathrm{~b})$, respectively. The dashed lines represent first order polynomial fits to the linear part of the curves. 
Table 3 show the results of the Pearson's $X^{2}$ test with York's weights of the data from Fig. 11 inserted in Eq. (9). As expected, the correlation showed a significant improvement compared with Tab. 2, especially in the case of pure $\mathrm{CO}_{2}$ where the test now was within a $90 \%$ confidence interval. This indicates that the $\delta T_{\mathrm{e}}$ and $T_{\mathrm{e}}$ depended similarly on the vibrational excitation of $\mathrm{CO}_{2}$ from electron collisions, whereas the collisional change in electron density did not normalize the increased OG signal. However, even though the relationship between $U_{\mathrm{OG}}$ and $U_{\mathrm{P}}$ showed better agreement, the collisional excitation still had a significant effect on the OG signal, and a correction for such mechanisms needs to be incorporated into the model if it is to become truly accurate for $\mathrm{CO}_{2}$ plasmas in this particular regime. Nonetheless, the fact that a model based on such substantial simplifications as the one presented here, still could predict the mechanisms behind the generation of the OG effect with surprising accuracy, shows great potential for further development.

TAB. 3. Goodness of fits of data from Figs. 7 and 11 to Eq. (9), evaluated with Pearson's $X^{2}$ test with York's weights.

\begin{tabular}{ccc}
\hline$F(x)$ & Gas & $X^{2}$ \\
\hline$\frac{\partial U_{O G}}{\partial U_{\mathrm{P}}}\left(\frac{e \gamma^{2}}{k_{\mathrm{B}} T_{\mathrm{e} 0} p}\right)$ & $\begin{array}{c}0.25 \% \mathrm{CO}_{2} \\
\text { in } \mathrm{N}_{2}\end{array}$ & 0.87 \\
$\frac{\partial U_{O G}}{\partial U_{\mathrm{P}}}\left(\frac{e \gamma^{2}}{k_{\mathrm{B}} T_{\mathrm{e} 0} p}\right)$ & $\mathrm{CO}_{2}$ & 1.45 \\
\hline
\end{tabular}

With the results of Tabs. 2 and 3 at hand, it was possible to rewrite the OG proportionality parameter, $K$, of Eq. (1) in terms of the plasma parameters so that:

$$
\left\{\begin{array}{l}
\frac{\partial U_{\mathrm{OG}}}{\partial U_{\mathrm{B}}} \approx \pi n_{\mathrm{m}} I_{0} \sigma_{\mathrm{L}} l r^{2} K_{0}\left(\frac{e}{k_{\mathrm{B}} T_{\mathrm{e} 0}}+\frac{\alpha}{U_{\mathrm{PP} 0}}\right) \\
\frac{\partial U_{\mathrm{OG}}}{\partial U_{\mathrm{P}}} \approx \pi n_{\mathrm{m}} I_{0} \sigma_{\mathrm{L}} l r^{2} \frac{e K_{0}^{\prime}}{k_{B} T_{e 0}}
\end{array} \text { for } U_{\mathrm{PP} 0}<U_{\mathrm{B}}<U_{\mathrm{Q}},\right.
$$

where $K_{0}$ and $K_{0}$ ' now are constants. Hence, it can be expected that a colder plasma yields a stronger OG signal, which agrees with experimental observations. Remembering the asymmetric distribution of the plasma in the gap (inset of Fig. 1), this temperature dependence highlights an advantage of the SSRR measurement scheme, where the OG signal is measured at the probe with the lowest electron temperature [15]. However, it should be remembered that Eq. (10) is limited to a confined interval of $U_{\mathrm{B}}$, and also to electron temperatures where electron-molecule collisions do not strongly affect the number of molecules susceptible to the laser beam. Still, within this region, it should be possible to find optimum operation conditions for the system in terms of, e.g., pressure, microwave power, biasing voltage, etc.

Interesting with respect to spectrometry applications was the dependency of $U_{\mathrm{OG}}\left(U_{\mathrm{P}}\right)$ on the pressure in the sample cell, Fig. 12. As can be seen, a clear linear dependence was found between the OG effect and the 
pressure in both experiments. Such a behavior is expected from a well-behaved OG system (see, e.g., [32]), which once more highlights the great potential of the SSRR concept for both OG spectroscopy and spectrometry, although further investigations are required to evaluate, e.g, the sensitivity, dynamic range and detection limit of the system.

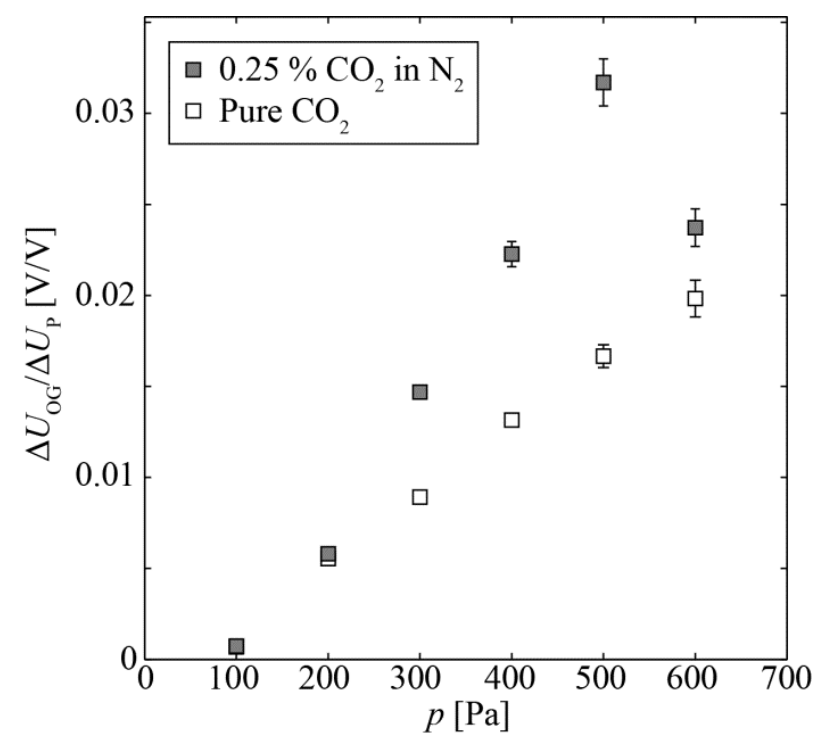

FIG. 12. First order derivative of the OG signal with respect to $U_{\mathrm{P}}$, for gas mixes of $0.25 \% \mathrm{CO}_{2}$ in $\mathrm{N}_{2}$ (gray squares) and pure $\mathrm{CO}_{2}$ (white squares) at different pressures. Error bars correspond to $95 \%$ confidence bounds.

Finally, the stability of the OG signal at different bias voltages was investigated by studying the overlapped Allan variance. Figure 13 show the results for two different pressures of pure $\mathrm{CO}_{2}$. Before the calculation, the signal was normalized to the global mean value of the data set. As can be seen, the stability improved as the bias voltage increased, yielding a maximum integration time, $\tau$, of $60-70 \mathrm{~s}$ at $U_{\mathrm{B}}=50 \mathrm{~V}$. This constituted a $\sim 40 \%$ improvement compared with the unbiased case [16]. At negative bias, however, the stability deteriorated compared with the unbiased case, likely due to the drift observed in Fig. 5. The drift was, in turn, possibly caused by heating of probe 1 due to ion bombardment, and, eventually, it would possibly stabilize, although no immediate reasons to utilize negative bias was found in this study. 


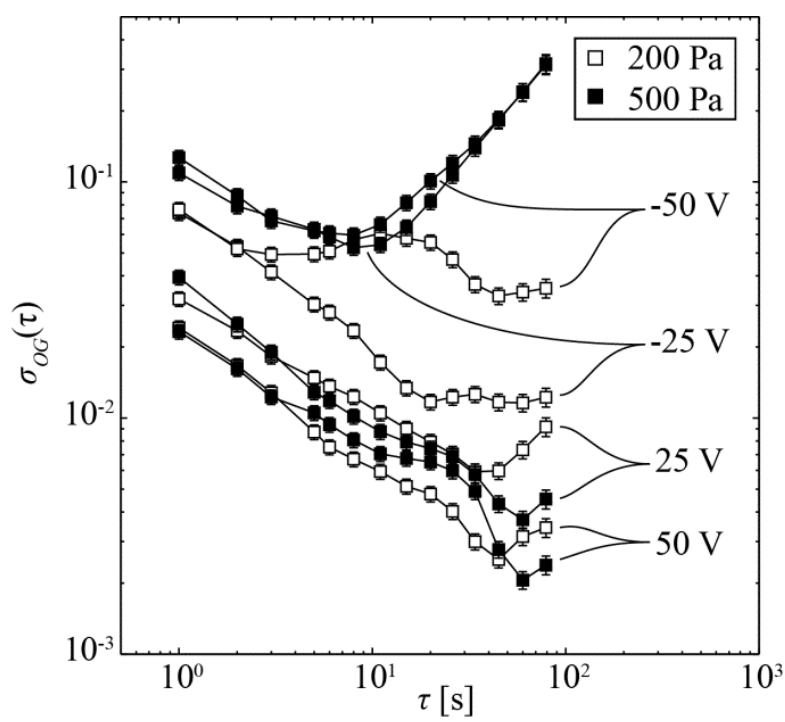

FIG. 13. Allan variance of the normalized OG signal with different probe bias at $200 \mathrm{~Pa}$ (white squares) and 500_Pa (black squares), respectively.

\section{Conclusion}

It has been shown that applying a constant bias voltage to one of the probes in a SSRR effectively improved the performance of the detector, by increasing both the amplitude and the stability of the OG signal. More precisely, the SNR increased linearly by about one order of magnitude in a voltage interval between the equilibrium plasma potential and a point where the probe biasing started to quench the plasma, roughly between 20 and $60 \mathrm{~V}$. Simultaneously, the stability was improved by about $40 \%$ compared with previous studies [16].

Furthermore, it was shown that relatively straightforward plasma theory could be applied to interpret the mechanism behind the generation of the OG signal in an SSRR detector. When the plasma was biased with a voltage above the equilibrium plasma potential, it was shown that the OG signal became a function of the electron density and energy. The proposed theoretical model allowed for better correlation between the OG signal and the number of target molecules in the sample, which is of great importance in spectrometric applications.

However, in a pure $\mathrm{CO}_{2}$ plasma with electron energies in the $1-6 \mathrm{eV}$ range, excitation of $\mathrm{CO}_{2}$ to the first symmetric stretch mode by electron-molecule collisions was shown to be an important process in the generation of the OG signal. Such non-linear processes were not included in the model, but demonstrated that further studies are required to fully understand the OG signal generation in the SSRR. Moreover, in situ monitoring and control of important input parameters, such as the pressure, temperature and possibly flow of the sample gas, will become important in an actual application. Efforts to integrate such sensors in the sample cell of the SSRR are currently being made. 
Regardless of these improvements, the SSRR concept already shows exciting performance, especially given its uncomplicated fabrication, compact size, and straightforward signal detection. With respect to cavityenhanced IR spectroscopy, the drive for longer effective path lengths constantly increases the requirements on the detector, which, in the case of absorption detectors, becomes increasingly expensive and difficult to operate. An SSRR detector would, on the other hand, only benefit from longer effective path lengths, and should therefore evolve into a compact, inexpensive and easy-to-operate alternative for future IR spectrometers.

\section{Acknowledgment}

The authors wish to acknowledge the Swedish Research Council for supporting this project (Ref. A0442201). Assoc. Prof. Greger Thornell and Prof. Göran Possnert, Uppsala University, is gratefully acknowledged for their contributions.

\section{References}

[1] Y. Yao, A. Hoffman, and C. Gmachl, "Mid-infrared quantum cascade lasers," Nature Photonics, vol. 6, pp. 432-439, 2012.

[2] I. Galli, S. Bartalini, S. Borri, P. Cancio, D. Mazzotti, P. De Natale, and G. Giusfredi, "Molecular Gas Sensing Below Parts Per Trillion: Radiocarbon-Dioxide Optical Detection," Physical Review Letters, vol. 107, p. 270802, 2011.

[3] V. M. Baev, T. Latz, and P. E. Toschek, "Laser intracavity absorption spectroscopy," Applied Physics $B$, vol. 69, pp. 171-202, 1999/09/01 1999.

[4] J. H. van Helden, N. Lang, U. Macherius, H. Zimmermann, and J. Röpcke, "Sensitive trace gas detection with cavity enhanced absorption spectroscopy using a continuous wave external-cavity quantum cascade laser," Applied Physics Letters, vol. 103, p. 1311142013.

[5] H. Jane and P. T. Ralph, "Optical gas sensing: a review," Measurement Science and Technology, vol. 24, p. 012004, 2013.

[6] J. B. Paul, L. Lapson, and J. G. Anderson, "Ultrasensitive absorption spectroscopy with a high-finesse optical cavity and off-axis alignment," Applied Optics, vol. 40, pp. 4904-4910, 2001/09/20 2001.

[7] C. Dyroff, "Optimum signal-to-noise ratio in off-axis integrated cavity output spectroscopy," Optics Letters, vol. 36, pp. 1110-1112, 2011/04/01 2011.

[8] B. Barbieri, N. Beverini, and A. Sasso, "Optogalvanic spectroscopy," Reviews of Modern Physics, vol. 62, pp. 603-644, 1990. 
[9] A. Rogalski, "History of infrared detectors," Opto-Electronics Review, vol. 20, p. 279, 2012.

[10] X. Yuan, J. Tang, and Y. Duan, "Microplasma Technology and Its Applications in Analytical Chemistry," Applied Spectroscopy Reviews, vol. 46, pp. 581-605, 2011/10/012011.

[11] M. J. Madou, Fundamentals of Microfabrication: The Science of Miniaturization, Second Edition: Taylor \& Francis, 2002.

[12] M. G. Kong, G. Kroesen, G. Morfill, T. Nosenko, T. Shimizu, J. v. Dijk, and J. L. Zimmermann, "Plasma medicine: an introductory review," New Journal of Physics, vol. 11, p. 115012, 2009.

[13] D. E. Murnick, O. Dogru, and E. Ilkmen, "Intracavity Optogalvanic Spectroscopy. An Analytical Technique for 14C Analysis with Subattomole Sensitivity," Analytical Chemistry, vol. 80, pp. 48204824, 2008.

[14] D. E. Murnick and B. J. Peer, "Laser-based analysis of carbon isotope ratios," Science, vol. 263, pp. 945-947, February 18, 19941994.

[15] T. Suzuki, H. Sekiguchi, and T. Kasuya, "Optogalvanic detection with microwave discharge," Journal de Physique, vol. C7-44, p. 419, 1983.

[16] A. Persson, M. Berglund, G. Thornell, G. Possnert, and M. Salehpour, "Stripline split-ring resonator with integrated optogalvanic sample cell," Laser Physics Letters, vol. 11, p. 045701, 2014.

[17] M. Berglund, M. Grudén, G. Thornell, and A. Persson, "Evaluation of a microplasma source based on a stripline split-ring resonator," Plasma Sources Science and Technology, vol. 22, p. 055017, 2013.

[18] M. Berglund, G. Thornell, and A. Persson, "Microplasma source for optogalvanic spectroscopy of nanogram samples," Journal of Applied Physics, vol. 114, p. 0333022013.

[19] G. Eilers, A. Persson, C. Gustavsson, L. Ryderfors, E. Mukhtar, G. Possnert, and M. Salehpour, "The radiocarbon intracavity optogalvanic spectroscopy setup at Uppsala," Radiocarbon, vol. 55, pp. 237250, 2013.

[20] A. Persson, G. Eilers, L. Ryderfors, E. Mukhtar, G. Possnert, and M. Salehpour, "Evaluation of Intracavity Optogalvanic Spectroscopy for Radiocarbon Measurements," Analytical Chemistry, vol. 85, pp. 6790-6798, 2013/07/16 2013 .

[21] H. Sekiguchi, A. Masuyama, T. Kasuya, and T. Suzuki, "Investigation of the optogalvanic effect ina microwave discharge," Journal of Applied Physics, vol. 58, p. 154, 1985.

[22] C. R. Webster and R. T. Menzies, "Infrared laser optogalvanic spectroscopy of molecules," The Journal of Chemical Physics, vol. 78, pp. 2121-2128, 1983. 
[23] R. L. Merlino, "Understanding Langmuir probe current-voltage characteristics," American Journal of Physics, vol. 75, pp. 1078-1085, 2007.

[24] F. Czerwinski, "allan v1.71," MatlabCentral, vol. 21727, 2008.

[25] Y. Itikawa, "Cross Sections for Electron Collisions With Carbon Dioxide," Journal of Physical and Chemical Reference Data, vol. 31, pp. 749-767, 2002.

[26] B. Chapman, Glow discharge processes. New York: Wiley, 1980, pp. 64-65.

[27] W. T. Silfvast, Laser Fundamentals. Cambridge: Cambridge University Press, 1996.

[28] N. Miura and J. Hopwood, "Spatially resolved argon microplasma diagnostics by diode laser absorption," Journal of Applied Physics, vol. 109, pp. -, 2011.

[29] M. Krystek and M. Anton, "A weighted total least-squares algorithm for fitting a straight line," Measurement Science and Technology, vol. 18, p. 3438, 2007.

[30] G. Liakhou, S. Paoloni, and M. Bertolotti, "Observations of laser cooling by resonant energy transfer in CO2-N2 mixtures," Journal of Applied Physics, vol. 96, pp. 4219-4224, 2004.

[31] Y. Itikawa, "Cross Sections for Electron Collisions with Nitrogen Molecules," Journal of Physical and Chemical Reference Data, vol. 35, pp. 31-53, 2006.

[32] F. O. Shimizu, K. Sasaki, and K. Ueda, "Optogalvanic Effect Study of Vibrational Relaxation in CO 2 Laser Process," Japanese Journal of Applied Physics, vol. 22, p. 1144, 1983. 